\title{
Diabetes and Pregnancy
}

Authors

Helmut Kleinwechter ${ }^{1}$, Ute Schäfer-Graf², Christoph Bührer ${ }^{3}$, Irene Hoesli, Franz Kainer ${ }^{5}$, Alexandra Kautzky-Willer6 Brigitte Pawlowski ${ }^{7}$, Karl-Ulrich Schunck ${ }^{8}$, Thierry Somville ${ }^{9}$, Marianne Sorger ${ }^{10}$

\author{
Affiliations \\ 1 Diabetologikum Kiel, Diabetes Specialist Practice and \\ Training Center, Kiel, Germany \\ 2 Berlin Diabetes Center for Pregnant Women, Clinic for \\ Gynecology and Obstetrics, St. Joseph Hospital, Berlin, \\ Germany \\ 3 Department of Neonatology, Charité University \\ Medicine, Berlin, Germany \\ 4 Gynaecological Clinic, Obstetrics and Pregnancy \\ Medicine, University Hospital Basel, Basel, Germany \\ 5 Hallerwiese Clinic, Department of Obstetrics and \\ Prenatal Medicine, Nuremberg, Germany \\ 6 Medical University of Vienna, Clinic for Internal \\ Medicine III, Department of Endocrinology and \\ Metabolism, Vienna, Germany \\ 7 German Diabetes Clinic, German Diabetes Center, \\ Düsseldorf, Germany \\ 8 Clinic for Child and Youth Medicine, Hospital in \\ Friedrichshain, Berlin, Germany \\ 9 Department of Obstetrics and Prenatal Medicine, \\ University Medical Center Hamburg-Eppendorf, \\ Hamburg, Germany \\ 10 Medical Polyclinic, University Hospital Bonn, Bonn, \\ Germany
}

published online $\quad 08.01 .2021$

\section{Bibliography}

Exp Clin Endocrinol Diabetes 2021; 129: S1-S8

DOI $10.1055 / a-1284-5758$

ISSN 0947-7349

(C) 2021. Thieme. All rights reserved.

Georg Thieme Verlag KG, Rüdigerstraße 14,

70469 Stuttgart, Germany

German Diabetes Association: Clinical Practice Guidelines This is a translation of the DDG clinical practice guideline published in Diabetologie 2020; 15 (Suppl 1): S1-S8,

DOI 10.1055/a-1193-3815

\section{Correspondence}

AG Spokeswoman Gynecology and Obstetrics

Prof. Dr. med. Ute Schäfer-Graf

Berlin Diabetes Center for Pregnant Women

Clinic for Gynecology and Obstetrics

St. Joseph Hospital Wüsthoffstraße 15

12101 Berlin

Germany

ute.schaefer-graf@sjk.de

AG Speaker Internal Medicine and Diabetology

Prof. Dr. Michael Hummel

Diabetological SPP Rosenheim \& Diabetes Research Group

TU Munich

Max-Josefs-Platz 21

83022 Rosenheim

Germany

michael.hummel@Irz.uni-muenchen.de

\section{Preliminary Note}

This practice guideline addresses only type 1 and type 2 diabetes already diagnosed before pregnancy. These are high-risk pregnancies and require joint care by specialized diabetologists, obstetricians and neonatologists in close cooperation with midwives, ophthalmologists and other specialists.

\section{Prevalence}

In 2017, German maternity hospitals registered pre-conceptionally-diagnosed diabetes in $0.93 \%$ of about 761481 pregnancies $(n=7096)$. This prevalence increased by $5.2 \%$ compared to 2016 .
A differentiation into type 1 and type 2 diabetes is not possible from the available data.

The proportion of pregnant women with type 2 diabetes is estimated at about $20 \%$.

\section{Metabolic Targets}

A metabolic adjustment close to the norm should be achieved with an $\mathrm{HbA} 1 \mathrm{c}<7 \%$ (better $<6.5 \%$ ) for at least 3 months before conceiving. The blood glucose target values (capillary blood measurements using the patient's meter) after the beginning of the pregnancy are compiled in > Table 1. 
> Table 1 Blood glucose target values (capillary measurement as plasma equivalent) after the start of pregnancy.

\begin{tabular}{|l|l|l|}
\hline Time & $\mathbf{~ m g / d ~}$ & $\mathbf{m m o l} / \mathbf{l}$ \\
\hline Fasting, preprandial & $65-95$ & $3.6-5.3$ \\
\hline 1 h postprandial & $<140$ & $<7.7$ \\
\hline 2 h postprandial & $<120$ & $<6.6$ \\
\hline Before bed & $90-120$ & $5.0-6.6$ \\
\hline At night 2:00-4:00 & $>65$ & $>3.6$ \\
\hline Medium blood glucose (MBG) & $90-110$ & $5.0-6.1$ \\
\hline
\end{tabular}

The mean blood glucose (MBG) values of one day, consisting of 6 values (before main meals and $1-2 \mathrm{~h}$ afterwards), of $<90 \mathrm{mg} / \mathrm{dl}$ $(5.0 \mathrm{mmol} / \mathrm{l})$ indicate too narrow a setting with the risk of fetal growth retardation, MBG values of $>110 \mathrm{mg} / \mathrm{dl}(6.1 \mathrm{mmol} / \mathrm{l})$ are considered to be insufficiently well-adjusted. The postprandial values (after 1 or $2 \mathrm{~h}$ ) are important for fetal prognosis, after which the subsequent preprandial insulin doses should be adjusted.

Immediate postprandial correction should be made as of $200 \mathrm{mg} / \mathrm{dl}(11.0 \mathrm{mmol} / \mathrm{l})$. The $\mathrm{HbA} 1 \mathrm{c}$ value should be determined every 4-6 weeks and should be within the reference range for healthy individuals. (Different regional reference ranges of the $\mathrm{HbA} 1 \mathrm{c}$ methods should be pointed out. In general, the HbA1c value before conceiving should be no more than $0.5-1 \%$ absolute above the upper reference limit of the laboratory method used).

The quality of blood glucose self-monitoring by the pregnant woman should be checked regularly with device-specific control solutions.

\section{CONCEPTT Study}

The blood glucose targets required by the guidelines are often not achieved in pregnant women with type 1 diabetes, and the rate of malformations, complications and LGA children is therefore unfortunately still significantly increased. Is the Continuous Glucose Monitoring System (CGMS) an option to improve glucose control and reduce complications? So far, 3 randomized CGMS studies have provided an answer to this question, but the data are mixed. Two studies include type 1 diabetes and type 2 diabetes, in some cases only an intermittent masked CGMS was used and the case numbers were small, so that in 2017, a Cochrane review concluded that the evidence is small and a large RCT is needed. In 2017, the CONCEPTT study was published in the Lancet (Feig DS, Donovan LE, Corcoy R et al. Continuous glucose monitoring in pregnant women with type 1 diabetes (CONCEPTT): a multicentre international randomised controlled trial. Lancet 2017; 390: 2347-2359), which addresses this issue. Inclusion criteria were type 1 diabetes for at least 1 year, age between $18-40$ years and suboptimal glycemic control. The primary endpoint was the change in $\mathrm{HbA} 1 \mathrm{c}$ in the 34th week of pregnancy, secondary endpoints were the results of CGM measurements, the neonatal outcome and other endpoints such as preterm rupture of the membranes. Using CGM, pregnant women with type 1 diabetes were able to reduce their HbA1c levels $0.2 \%$ more than with conventional blood glucose monitoring and, 34 weeks later, were in the glycemic target range for longer (time-in-range). These effects were not observed in women planning to become pregnant. Infants of CGM mothers showed a reduced risk of LGA $(N N T=6)$, neonatal intensive care admissions for $>24 \mathrm{~h}(\mathrm{NNT}=6)$, neonatal hypoglycemia with intravenous dextrose administration $(\mathrm{NNT}=8)$, and a one day reduced hospital stay. With CGM, pregnant women at week 34 spent approximately 100 min longer daily in the glucose target range. However, the authors' recommendation to consider CGMS as the standard for type 1 diabetes pregnancy seems premature. It would be important to identify patients who would particularly benefit from CGMS technology. Also, LGA rates in this study remained unusually high.

\section{Counseling for Fertility}

\section{Human genetic counseling}

The risk of children developing type 1 diabetes is $0.8 \%$ after 5 years ( $5.3 \%$ after 20 years). If the father also has type 1 diabetes, the 5 -year risk is $11 \%$. If a sibling is also affected in addition to the mother, the rate is $12 \%$ after 5 years. The risk in the general population is about $0.3 \%$ until the age of 25 .

\section{Abortion and malformation risk}

The risk for early abortions is increased and depends on the preconceptional metabolic control. Numerical chromosomal anomalies are not more frequent in diabetic pregnancies.

The risk of malformations also depends on the quality of the pre-conceptional metabolic control, it is on average about 4 times higher compared to the general population, on average $8.8 \%$. This applies equally to type 1 and type 2 diabetes. In planned pregnancies, the risk of malformations is lower than in unplanned pregnancies due to targeted counseling, better metabolic control and blood glucose self-monitoring. Malformations mainly affect the heart and vessels near the heart (risk: 4-fold), neural tube defects (risk: 2 to 3 -fold) and multiple malformations. There is no diabetes-specific type of malformation. The frequently-mentioned caudal regression syndrome is very rare (prevalence in diabetic pregnancies: $1.3 / 1000)$. The risk of malformations is also associated with obesity, microvascular complications and inadequate periconceptional folic acid substitution in the pregnant woman.

\section{Risk of perinatal mortality and infant death in the first year of life}

The risk of perinatal mortality in fetuses or neonates without malformations increases significantly above a periconceptional HbA1c of $6.6 \%$. This also applies to infant death until the end of the first year of life.

\section{Possibilities of reproductive medicine}

Fertility in women with well-adjusted diabetes is hardly reduced compared to metabolically healthy women. If the desire to have children is not fulfilled, there are no restrictions on the methods of infertility treatment that can be considered - after completion of the reproductive medical diagnostics in a specialized center. Cycle disorders are often normalized by an optimized insulin therapy.

\section{Folic acid and iodide substitution}

Periconceptional substitution with $0.4-0.8 \mathrm{mg}$ folic acid/day, starting at least 4 weeks pre-conception and until the end of week 12 (to prevent neural tube malformations and cleft lip and palate - if 
possible combined with a folate-rich diet), can be recommended to all pregnant women who wish to have children. Due to the increased incidence of diabetes, pregnancy and thyroid diseases and to ensure sufficient iodine supply to the fetus, medicinal iodine prophylaxis with at least $200 \mu \mathrm{g}$ iodide per day, information on iodine-rich foods and the use of iodized table salt are recommended (TSH screening of pregnant women, see below).

\section{Insulin therapy}

Insulin is the only well-studied pharmacotherapy for pregnancies with manifest diabetes. Pregnant women with type 2 diabetes must therefore be switched from oral antidiabetics to insulin either preconception or immediately after the diagnosis of pregnancy if a diet without rapidly resorbable carbohydrates is not sufficient. Intensified conventional insulin therapy (ICT) or, if indicated, continuous subcutaneous insulin infusion (CSII) by means of an insulin pump are the insulin strategies of choice and equivalent in terms of outcome.

Postprandial blood glucose levels are of particular importance for fetal growth, birth weight and thus perinatal risks. The strict, normal setting is limited by the maternal risk of hypoglycemia. Therefore, individual target agreements must be made in the case of frequent severe hypoglycemia, special life circumstances and a problematic social environment.

In the first trimester of pregnancy, the risk of maternal hypoglycemia increases - adverse effects on embryogenesis are not known, but long-term follow-up observations of the children regarding their psychomotor development are lacking. The insulin requirement increases continuously starting in the 2 nd trimester of pregnancy ( + 50-100\% until birth) and can be extremely high, especially in obese pregnant women with type 2 diabetes, while the risk of hypoglycemia decreases at the same time. At the time of birth, the insulin requirement drops relatively quickly. At the beginning of labor, the basal insulin requirement is reduced by about $50 \%$ (e. g. below CSII). During labor, only short-acting insulin is administered, also intravenously, depending on local practice. Postpartum, insulin substitution is individually readjusted within a few days; the pre-conceptional need serves as a guide.

\section{Choice of insulin preparation}

Human insulin is the medication of choice. Pregnant women who are well-adjusted to the short-acting insulin aspart or lispro insulin analogues can continue to use them. There is no experience with insulin glulisin and insulin degludec. Women who are well-adjusted to the long-acting insulin analogue insulin detemir can also continue to use it. Insulin glargin can continue to be used if targets are well-set before conceiving.

If the metabolic control is optimal during early pregnancy (HbA1c $<7 \%$, no frequent hypoglycemia), a change of insulin preparations or therapeutic strategies (e. g. CSII for ICT) must be weighed against the risk of a possible metabolic deterioration.

\section{Diabetological emergencies}

It is imperative that pregnant women avoid severe hypoglycemia with the need for glucose or glucagon injections. A stable, nearnormal setting can result in hypoglycemic warning signs increas- ingly being suppressed and eventually disappearing completely due to insufficient hormonal counter-regulation. The partner or another relative must be informed about hypoglycemia and instructed in the use of the emergency glucagon kit. Ketoacidosis in diabetic pregnancy is a critical emergency situation. Immediate admission to a suitable clinic with emergency medical supervision is indicated. There, coma therapy should be started immediately according to the in-house treatment plan and the further procedure should be carried out in close consultation with diabetologists, obstetricians and neonatologists.

\section{Complications and Concomitant Diseases}

\section{Arterial hypertension}

A distinction must be made between hypertensive forms with preconceptional therapy and pregnancy-specific hypertensive diseases (gestational hypertension, pre-eclampsia), which only manifest themselves after the 20th week of pregnancy. The correct blood pressure measurement during pregnancy should be observed ( $\triangleright$ Table 2). While the blood pressure therapy goal before conceiving and up to the 20th week of pregnancy is $<140 / 90 \mathrm{mmHg}$, the threshold for the initial blood pressure intervention in pregnancy is higher after the 20th week: therapy is only started as of values of $160 / 100 \mathrm{mmHg}$ as there is a risk of fetal growth retardation if therapy is started too early. Clinical symptoms of pre-eclampsia require therapy at lower blood pressure levels. The primary management of pregnancy-specific high-pressure therapy after the 20th week is the responsibility of the obstetrician in close consultation with the diabetologist. In diabetic nephropathy, individual therapy goals are given priority, the risk of pre-eclampsia is reduced by tight blood pressure control of $<140 / 90 \mathrm{mmHg}$ before conceiving and before the 20th week of pregnancy.

- Pregnancy per se (hormonal change)

- Unfavorable pre-conception initial situation

- Insufficient pre- conception laser coagulation

- Arterial hypertension

- Diabetic nephropathy

- Smoker

- High periconceptional HbA1c level

- Diabetes duration> 10 years

- Anemia

ACE inhibitors, AT1 receptor inhibitors and the direct renin inhibitor aliskiren are contraindicated throughout pregnancy and must be converted before conceiving - alpha-methyldopa is the medication of choice. If alpha-methyldopa is not sufficient as monotherapy, it can be combined with cardio-selective beta-receptor blockers (e.g. metoprolol) and calcium channel blockers (e.g. nifedipine "off-label"). Atenolol should not be used because of the risk of growth retardation. Due to the possibility of neonatal bradycardia with beta-blocker therapy, the neonatologist should be informed antenatally. Diuretics should not be restarted during pregnancy; diuretic therapy that has already been started pre-conceptionally can be continued; thiazide diuretics can make a normoglycemic adjustment difficult - a therapy adjustment may be necessary. 
- Table 2 Notes on correct blood pressure measurement for pregnant women.

\begin{tabular}{|l|}
\hline 5 min of rest before taking the blood pressure measurement \\
\hline $\begin{array}{l}\text { Mercury sphygmomanometers with upper arm measurement are } \\
\text { preferable }\end{array}$ \\
\hline Observe proper cuff width (depends on the manufacturer) \\
\hline $\begin{array}{l}\text { - Standard cuff } 12-13 \mathrm{~cm} \text { wide and } 35 \mathrm{~cm} \text { long for arm circumfer- } \\
\text { ence of } 24-32 \mathrm{~cm}\end{array}$ \\
\hline - Cuff $15 \times 30 \mathrm{~cm}$ for arm circumference $33-41 \mathrm{~cm}$ (thick arms) \\
\hline - Cuff $18 \times 36 \mathrm{~cm}$ for arm circumference $>41 \mathrm{~cm}$ (very thick arms) \\
\hline - Cuff $10 \times 18 \mathrm{~cm}$ for arm circumference $<24 \mathrm{~cm}$ (very thin arms) \\
\hline Pregnant woman sits or lies with upper body raised at a $45^{\circ}$ angle \\
\hline Remove all clothing from the arm \\
\hline Place cuff at heart level for blood pressure measurement \\
\hline At the first measurement, compare right and left sides, use higher value \\
\hline Measure blood pressure accurately to the nearest 2 mmHg \\
\hline $\begin{array}{l}\text { At the first measurement, perform at least two measurements at } \\
\text { intervals of } 1-2 \text { min }\end{array}$ \\
\hline $\begin{array}{l}\text { In case of increased values, confirm by a second measurement after at } \\
\text { least } 4 \mathrm{~h}\end{array}$ \\
\hline Systolic blood pressure $\rightarrow$ Korotkoff phase I (= Faint tapping sound) \\
\hline Diastolic blood pressure $\rightarrow$ Korotkoff phase $\mathrm{V}(=$ silence) \\
\hline
\end{tabular}

Other than magnesium sulfate as the basic therapy, the frequently intravenously-administered dihydralazine is no longer the drug of first choice due to its complication rates at higher doses for the treatment of hypertensive emergencies and impending eclampsia. Nifedipine and urapidil are the preferred alternatives. A combination of magnesium and nifedipine can cause a greater drop in blood pressure due to the potentiating effect of both substances.

\section{Diabetic retinopathy}

This is the most common microvascular complication in diabetic pregnant women and can first manifest itself during pregnancy. Existing lesions may worsen during pregnancy, usually in the 3rd trimester. Risks of progression are:

In the absence of diabetic retinopathy, 4 ophthalmologic examinations with dilated pupils are indicated:

- Pre-conception in pregnancy planning

- Immediately after diagnosis of pregnancy

- Every 3 months until birth

In case of already diagnosed retinopathy or new manifestation, individual controls are agreed upon by the ophthalmologist (who must be a retinal specialist). If an active, proliferative retinopathy exists in the case of infertility (possibly with insufficient metabolic control), the complete regression of the retinal findings should be awaited after adequate laser coagulation and the metabolism should be lowered to the required target range before planning to conceive.

Diabetic retinopathy is not per se an indication for C-section delivery. If retinopathy is known, multiple individual controls are indicated in the 1st year after delivery.

\section{Diabetic nephropathy}

Pre-conception stage classification should be based on the new clinical nephropathy classification (see Nephropathy Practice Guideline). Pregnancy per se does not lead to a decrease in GFR after birth.

After the diagnosis of pregnancy, an albumin screening should be performed at the beginning of each trimester, even if the results are negative. The screening for albuminuria (mean value from 2 spontaneous urine samples due to high variability) correlates well in pregnant women with albuminuria with the urine collected over $24 \mathrm{~h}$ (spontaneous urine: microalbuminuria $20-200 \mathrm{mg} / \mathrm{l}$, macroalbuminuria > $200 \mathrm{mg} /$ /; 24-h-urine: microalbuminuria 30-300 mg/ $24 \mathrm{~h}$, macroalbuminuria $>300 \mathrm{mg} / 24 \mathrm{~h}$ ) and, at its first appearance, indicates an increased risk of pre-eclampsia and preterm birth, among others. Due to the possibility of false negative and false positive results when measuring albumin in spontaneous urine without urinary creatinine, simultaneous measurement of this value is recommended (women: microalbuminuria $30-300$ mg/g creatinine, macroalbuminuria: $>300 \mathrm{mg} / \mathrm{g}$ creatinine). False positive findings, e. g. during physical exertion, urinary tract infections or insufficiently-controlled diabetes must be taken into account.

Nephropathy stages 1a/1b are associated with increased rates of pre-eclampsia and preterm birth. This risk is reduced by adjusting blood pressure to target values $<140 / 90 \mathrm{mmHg}$. The rate of preterm births before the 34th week of gestation can also be reduced by treatment of normotensive pregnant women with type 1 diabetes and microalbuminuria with alpha-methyldopa.

Nephropathies in diabetic pregnancy from stage 3 according to $\mathrm{KDOQI}$ are rare, but associated with high fetal, neonatal and infantile risks (preterm birth rate, growth retardation, intrauterine death, perinatal/neonatal mortality, psychomotor retardation in childhood). There is also a significant maternal-social risk after birth: inclusion in the dialysis program, increased mortality before their children reach adulthood. Pregnancies in diabetic women during hemodialysis or peritoneal dialysis, as well as after a double kidney or kidney-pancreas transplant are very rare.

Especially high maternal and fetal risks should be pointed out in case of diabetic nephropathy and the desire for children with:

- A serum creatinine level of $1.5 \mathrm{mg} / \mathrm{dl}$ ( $133 \mu \mathrm{mol} / \mathrm{l}$ and above),

- Nephropathy from stage 3 (creatinine clearance $<60 \mathrm{ml} /$ $\min \times 1.73 \mathrm{~m}^{2}$ )

- Arterial hypertension that is difficult to control

An individual risk assessment in cooperation with the nephrologist is essential.

\section{Diabetic neuropathy}

In the case of planned pregnancy and a diabetes duration $>10$ years, it should be checked whether there are indications of diabetic gastroparesis, orthostatic hypotension or hypoglycemia perception disorders. Pregnancy per se does not lead to the recurrence or progression of neuropathic changes.

\section{Macroangiopathy}

Women with diabetes have a 3-fold increased cardiovascular risk, which is further increased by pregnancy. At risk are women who 
are older, with a long diabetes duration, nephropathy, arterial hypertension and who are smokers. After a myocardial infarction, women should wait at least one year before becoming pregnant. An individual risk analysis in cooperation with the cardiologist is imperative.

\section{Thyroid}

There is a high prevalence of autoimmune thyroiditis in diabetic women, especially those over 30 years of age. Before conceiving or when pregnancy is diagnosed, TSH should be determined. If the TSH level is elevated, FT4 and TPO should be determined and a thyroid sonography should be performed. Subclinical hypothyroidism must be substituted with L-thyroxine. Because of the increased risk of postpartum thyroid dysfunction, laboratory tests (TSH) should be performed within 3-12 months after birth. lodide administration during and after pregnancy can also be carried out in the case of an increased TPO titer. Autoimmune thyroiditis of the Hashimoto type is thereby neither induced nor aggravated.

In cases of manifest hyperthyroidism in pregnancy, low doses of thiamazole or propylthiouracil can be used as monotherapy.

Thyrostatic drugs are harmless even in low doses during breastfeeding. lodide is contraindicated in hyperthyroidism.

\section{Examinations to Diagnose Fetal Condition}

\section{Ultrasound examinations}

The maternity guidelines provide for 3 ultrasound examinations, which must be supplemented by additional examinations in diabetic pregnant women:

- 8th-12th week: physical integrity of pregnancy and heart movement

- 11th-14th week: nuchal translucency measurement (optional)

- 19-22nd week: differentiated organ diagnostics (according to Level DEGUM II)

- From 24th week: biometrics every 2-4 weeks, more often in case of abnormalities

Ultrasound signs for diabetes-specific macrosomia are:

- Increase in abdominal circumference $>75$ th percentile (according to Hadlock) with normal growth of head and femur

- Abdominal circumference considerably larger than the head circumference in relation to week of gestation

Before delivery, an estimation of the birth weight and assessment of the ratio of abdomen (insulin-sensitive) to head (non-insulinsensitive) is recommended.

\section{Doppler ultrasound}

The indication for this examination is independent of the diagnosis of diabetes in pregnant women. It is an additional monitoring method in case of growth retardation of the fetus $>$ Table 3.

\section{Cardiotocography (CTG)}

The frequency of CTG controls should be adjusted to the individual fetal and maternal risks. This allows early detection of impend- ing intrauterine asphyxia, particularly with fetal risks such as growth retardation or macroscopic tendencies.

\section{Contraction stress test, biophysical profile, hormone determination, amniotic insulin, fetal movements}

The contraction stress test as well as the determination of the biophysical profile are obsolete as routine measures in diabetes. Measurements of estriol and placental lactogen are also obsolete. Amniotic fluid insulin measurements from the 28 th week of gestation are suitable for the quantification of fetal hyperinsulinism, but because of the invasiveness (amniocentesis required) and the fact that only a few centers offer fast and reliable laboratory tests, they are not a routine method and are therefore reserved for individual cases.

In some cases (e.g. in rural areas), the self-monitoring of fetal movements by the pregnant woman by means of daily counts ("count to ten") can help to improve the early detection of fetal risks in the home environment if ultrasound/Doppler ultrasound and CTG are not available promptly or close by.

\section{Treatment of Obstetric Complications}

\section{Infections}

The frequency of urogenital tract infections in diabetic pregnant women is increased and associated with an increased rate of preterm births. Therefore, regular check-ups and early antibiotic therapy are recommended.

\section{Preterm birth}

Inhibiting contraction

Preterm contractions are treated with the aim of extending the gestation period until lung maturity is complete. Tocolysis over a longer period should be avoided. Oral tocolysis with a beta-mimetic and/or magnesium sulfate is ineffective. Due to the glycogenolytic effect and the frequent simultaneously-prescribed bed rest and glucocorticoid administration, IV tocolysis with a beta-mimetic leads to a considerable increase in blood glucose levels, so that insulin therapy must be adjusted at short notice: day $1+25 \%$, day 2 and $3+40 \%$, day $4+20 \%$, day $5+10-20 \%$. The additional insulin requirement is calculated on all days from the insulin requirement prior to steroid administration and includes all basal/bolus and correction administration (following the example of the National Hospital in Copenhagen). The oxytocin antagonist Atosiban is metabolically neutral and is considered to be the first choice as IV tocolytic.

\section{Inducing lung maturity}

The risk of preterm birth (birth before 37th week of gestation) is almost 5 times higher on average for diabetic mothers. At the same time, fetal hyperinsulinism inhibits surfactant formation resulting in the risk of respiratory distress syndrome in the newborn. In cases of imminent preterm birth before the 34th week of gestation, inducing fetal pulmonary maturation with $2 \times 12 \mathrm{mg}$ betamethasone every $24 \mathrm{~h}$ is indicated. After the start of glucocorticoid administration, an increase in the insulin requirement by $20-40 \%$ must be expected for 4 days. 
- Table 3 Action steps for the desire for children, pregnancy, birth.

\begin{tabular}{|c|c|}
\hline Time/event & What to do? \\
\hline \multirow[t]{14}{*}{ Desire to have children } & - Consultation with diabetologist and gynecologist \\
\hline & - Analyze accompanying risks \\
\hline & - Retinopathy (referral to ophthalmologist) \\
\hline & - Nephropathy (urinary albumin, serum creatinine, GFR according to MDRD equation) \\
\hline & - Neuropathy (anamnesis and clinical examination) \\
\hline & - Coronary heart disease (clinic, ECG, ergometry, echocardiography) \\
\hline & - $0.4-0.8 \mathrm{mg}$ folic acid/day, advice on folate-rich food \\
\hline & - Exchange oral antidiabetics for insulin \\
\hline & - Check training status, instruct relatives on glucagon set \\
\hline & - Check thyroid function with TSH screening \\
\hline & - Prescribe iodide $200 \mu \mathrm{g} /$ day, recommend iodized salt, nutritional advice \\
\hline & - Optimize metabolism (HbA1c<7\%) for at least 3 months \\
\hline & - Adjust high pressure therapy (exchange ACE inhibitors/AT-1 antagonists for alpha-methyldopa) \\
\hline & - Exchange glargine for NPH insulin \\
\hline \multirow[t]{4}{*}{ Pregnancy diagnosis } & - Consultation with diabetologist and gynecologist \\
\hline & - Information about blood glucose targets \\
\hline & - Ophthalmologisch examination \\
\hline & - Urinary albumin screening, then at the beginning of each trimester \\
\hline Every $4-8$ weeks & Check blood glucose self-monitoring device with control solution \\
\hline 8-12th week & Ultrasound - check the progress of the pregnancy \\
\hline 11-14th week & Ultrasound - optionally perform nuchal translucency measurement \\
\hline As of 16 th week & Adjust insulin dose as the need increases \\
\hline 19-22th week & Differentiated organ diagnostics (DEGUM Stage II - German Society for Ultrasound in Medicine) \\
\hline 20-24th week & Ophthalmological examination \\
\hline As of 24th week & Biometrics every $2-4$ weeks \\
\hline As of 32nd week & CTG control, frequency to be decided individually \\
\hline 32-36th week & Contact with perinatal center (at least LEVEL 2) \\
\hline 34-36th week & Ophthalmological examination \\
\hline 36-38th week & Estimate birth weight ( $>4500 \mathrm{~g}$ - discuss planned C-section) \\
\hline Early labor & Admission to the inpatient labor clinic, bed rest, tocolysis PO: nifidepin (off-label), i. v. Atosiban (therapy of choice) \\
\hline Immenent premature birth & Fetal lung maturation induction with $2 \times 12 \mathrm{mg}$ betamethasone over $24 \mathrm{~h}$, adjust insulin dose (+ $20-40 \%)$ \\
\hline \multirow[t]{2}{*}{$\begin{array}{l}\text { Gestational hypertension } \\
\text { Pre-eclampsia }\end{array}$} & $\begin{array}{l}\text { - ASS } 100 \mathrm{mg} / \text { day for prevention in high risks, high pressure therapy from } 160 / 100 \mathrm{mmHg} \text {, earlier for symptoms } \\
\text { (performed by perinatal center) }\end{array}$ \\
\hline & - Adequate monitoring \\
\hline Birth clinic & Timely appointment (at the latest in week 36 of pregnancy), for insulin therapy at the LEVEL 2 or LEVEL 1 perinatal center \\
\hline Birth & $\begin{array}{l}\text { Spontaneous delivery is aimed for, no further injections of long-acting insulin at the start of labor, continue to use } \\
\text { pump (basal rate to } 50 \% \text { ) }\end{array}$ \\
\hline Inducing & When the calculated delivery date is exceeded \\
\hline C-section Child & primary and secondary only from obstetrical indication \\
\hline \multirow[t]{4}{*}{ Kind } & - Planned and unplanned only from obstetrical indication \\
\hline & - Examination and evaluation by neonatologists within $24 \mathrm{~h}$ of birth, immediately in case of clinical abnormalities \\
\hline & - Immediate skin-to-skin breastfeeding with $30 \mathrm{~min}$ \\
\hline & - First blood glucose measurement after 2 h of life (see AWMF Guideline 024/006) \\
\hline Breastfeeding & Recommendation for 6-12 months, support in every respect \\
\hline Documentation & Document diabetes basic data and data on pregnancy/birth/newborn child \\
\hline
\end{tabular}

Maternal complications and emergencies - hypertension, pre-eclampsia/eclampsia

Pre-eclampsia is defined as an increase in blood pressure $>140 \mathrm{mmHg}$ systolic or $>90 \mathrm{mmHg}$ diastolic in combination with protein excretion $>300 \mathrm{mg} / 24 \mathrm{~h}$ after $20^{\text {th }}$ week of gestation. In gestational hy- pertension, proteinuria is absent. In severe pre-eclampsia, blood pressure values of $>160 \mathrm{mmHg}$ systolic and/or $>110 \mathrm{mmHg}$ diastolic are measured. In the case of such findings, the aim of intervention is to prevent eclampsia, which is characterized by the occurrence of cerebral seizures and the risk of intracerebral bleeding. 
The main symptom of HELLP syndrome, a typical complication of pre-eclampsia, is persistent right-sided upper abdominal pain in combination with specific changes in laboratory values: thrombocytopenia, hemolysis and increased transaminases. As part of primary prevention, by taking $100 \mathrm{mg}$ acetylsalicylic acid (ASA)/day, starting no later than the 16 th week of pregnancy, the risk of preeclampsia can be reduced in cases classified as high risk by obstetricians. In particular, these include pregnant women with type 1 diabetes with arterial hypertension and nephropathy. ASA should be discontinued before birth at approx. week 37 in accordance with the obstetrician instructions specific to the individual case. General primary prevention with low-dose ASA in all pregnant women with type 1 and type 2 diabetes can be advocated and is decided by the obstetrician. After the 20th week, high pressure therapy should be started as of $160 \mathrm{mmHg}$ systolic or $100 \mathrm{mmHg}$ diastolic. This can also be initiated earlier and with proper monitoring depending on clinical symptoms. The individual risk of pre-eclampsia can be assessed by Doppler ultrasound of the uterine artery ("Notch").

\section{Giving Birth}

\section{Choice of maternity clinic}

For all pregnant women with type 1 or type 2 diabetes (with insulin therapy), giving birth must be planned in a perinatal center of at least level 2 (guideline of the Joint Federal Committee of Physicians and Health Insurance Companies - Gemeinsamen Bundesausschusses der Ärzte und Krankenkassen). Early presentation at the clinic is mandatory, by week 36 at the latest. In the case of malformations diagnosed before birth, delivery must take place at a clinic with a direct connection to the corresponding surgical specialty area (pediatric, neurosurgery, cardiac surgery).

\section{Inducing birth and indication for C-section}

The indications for inducing labor correspond to those of metabolically healthy women. When the calculated date of delivery is reached, active birth management is indicated, exceeding the due date should be avoided. Early inducing as a routine method increases the $\mathrm{C}$-section rate without reducing neonatal risks. The mother's diabetes is not per se an indication for a planned C-section. If the estimated weight $>4500 \mathrm{~g}$ (for small women: $4250 \mathrm{~g}$ ), a planned C-section should be considered due to the increased risk of shoulder dystocia. For an estimated weight of 4000-4499 g: inform the pregnant women individually about the increased specific risk of shoulder dystocia according to fetal biometrics, especially in the case of a pronounced head-abdomen difference. The indication for an unplanned C-section should be encouraged in the event of obstructed labor or an abnormal CTG.

\section{Metabolic control during labor}

Blood glucose targets during labor should be $80-130 \mathrm{mg} / \mathrm{dl}$ (4.4$7.2 \mathrm{mmol} / \mathrm{l})$. Maternal hypo- or hyperglycemia as well as severe fluctuations in blood glucose should be avoided using a treatment plan with instructions for infusions and insulin dose adjustment. This plan must be present in the delivery room and must have been authorized by interdisciplinary, authorized internal staff and remains in effect until the umbilical cord has been cut. At the beginning of labor, only short-acting insulin should be injected instead of longacting insulin for better therapy control. The insulin pump can be used until the end of the labor with a lowered basal rate, even in the case of birth by C-section (attachment e. $g$. to the upper arm). During labor, hourly blood glucose checks are indicated for the pregnant woman (point-of-care test), from which immediate therapeutic consequences must be drawn. After the placenta is delivered, the insulin dose must be adjusted.

\section{Perinatal morbidity and mortality}

The preterm birth rate is 5 times higher (absolute: $25-58 \%$ ), depending on metabolic control. The transfer rate to the neonatal intensive care unit is also increased. The 7 infantile complications include hypoglycemia, hyperbilirubinemia, polycythemia, transient hypertrophic cardiomyopathy, respiratory disorders and convulsions.

The total perinatal mortality after the $22^{\text {nd }}$ week up to day 7 of life is not different for type 1 and type 2 diabetes (2.8\% and $2.5 \%$ ). However, the rates are 4 to 6 times higher than in the general population.

Etiological factors play a role:

- Malformations

- Birth complications

- Consequences of (sometimes iatrogenic) preterm birth

- Respiratory disorders

- Intrauterine death

\section{Postpartum and Postnatal Aspects}

In the first few hours after birth, no or very little insulin may be required. Approximately 3 days after birth, the insulin requirement is reduced by about $20 \%$ compared to before conceiving and the risk of hypoglycemia is increased. Blood glucose should be monitored every 4-6 $\mathrm{h}$ during this period to promptly detect trends in blood glucose development.

\section{Breastfeeding and vaccination of children of diabetic mothers}

Breastfeeding is strongly recommended for all mothers with diabetes. During breastfeeding, the insulin requirement may be reduced. Regular vaccinations of children do not increase their risk of type 1 diabetes. Children of mothers with type 1 diabetes are not at increased risk for beta-cell autoimmunity after supplementation with cow's milk formula compared to hydrolyzed infant formula.

\section{Special Features of Type 2 Diabetes}

Pregnancies in type 2 diabetes are on the rise. The perinatal risks are at least as high as for type 1 diabetes. Compared to type 1 diabetes, pregnant women with type 2 diabetes have a higher rate of obesity, a higher age, a shorter diabetes duration and a higher rate of concomitant risks and medication. They are more likely to belong to an ethnic minority. In addition, chronic arterial hypertension is more frequent, while pre-eclampsia is less frequent. Preparation before conceiving is worse and they take folic acid less frequently before conceiving. The first medical appointment is usually only after completion of embryogenesis, and a high per- 
centage are treated with oral antidiabetics during early pregnancy. Before conceiving, a high percentage of pregnant women with type 2 diabetes do not receive diabetic care, and in about $30 \%$ of cases, no HbA1c was documented 6 months prior to conceiving. The percentage of unplanned pregnancies is higher than for type 1 diabetes.

Type 2 diabetes in pregnant women should not be trivialized as the risks are comparable to type 1 diabetes. Training before conceiving, conversion from oral antidiabetics to insulin as well as diabetological co-supervision are necessary. The metabolic targets are similar to those of type 1 diabetes.

\section{Quality Control}

Pregnancies in which diabetes was already diagnosed beforehand are a rare event in a high-risk situation. The treatment quality of both outpatient and inpatient care for this target group should be centralized in the hands of specialized teams (competence centers). It should be obligatory to record pregnancies in which diabetes was already diagnosed in terms of process and outcome qual- ity and to compare these with formulated health objectives. Structured questionnaires should be made available for this purpose by the German Diabetes Society (Deutsche Diabetes Gesellschaft) and the German Society for Gynecology and Obstetrics (Deutsche Gesellschaft für Gynäkologie und Geburtshilfe).

\section{INFORMATION}

\section{Addresses on the Internet}

www.deutsche-diabetes-gesellschaft.de

- Detailed version of the evidence-based guidelines www.diabetes-deutschland.de

- Information platform on diabetes mellitus

\section{Conflict of Interest}

The authors declare that they have no conflict of interest. 